\title{
Hospital Blood Bank
}

National Cancer Institute

\section{Source}

National Cancer Institute. Hospital Blood Bank. NCI Thesaurus. Code C133264.

A hospital (or facility located within a hospital) that routinely collects or processes whole blood or blood components. 